\title{
Pure robotic extended pyelolithotomy: cosmetic replica of open surgery
}

\author{
Rishi Nayyar · Pankaj Wadhwa $\cdot$ Ashok K. Hemal
}

Received: 24 March 2007/ Accepted: 20 August 2007/Published online: 15 September 2007

(C) Springer London 2007

\begin{abstract}
Percutaneous nephrolithotomy (PCNL) has replaced open pyelolithotomy as the procedure of choice for treating large-burden renal stone disease, especially staghorn calculi. Although it is a minimally invasive procedure, it involves transgressing the renal parenchyma and is thus associated with its unique set of complications. The evolution of laparoscopic pyelolithotomy and robotic assistance has provided an opportunity to the surgeon to revisit pyelolithotomy in a minimally invasive manner following the age-old principles of the era of open renal surgery. We report the feasibility and our experience with this technique in three cases of partial staghorn calculus with intra-renal pelvis.
\end{abstract}

Keywords Urolithiasis - Staghorn calculus ·

Laparoscopy $\cdot$ Robotic $\cdot$ Pyelolithotomy

\section{Introduction}

With the wide availability and demonstrable efficacy of endourological techniques, the role of open surgery for renal stone disease has diminished in urological practice. Peculiarly, the earlier teaching of "intact stone removal" to ensure clearance and thereby hopefully preventing residual stones has been relegated to oblivion, replaced by newer technologies, each bettering the other in fragmenting the stone to

\footnotetext{
R. Nayyar · P. Wadhwa · A. K. Hemal (ه)

Department of Urology,

All India Institute of Medical Sciences,

New Delhi 110029, India

e-mail: akhaiims@gmail.com

R. Nayyar

e-mail: nayyarrishi@gmail.com
}

"bits". However, with the development of laparoscopy, the principles of open renal surgery is being revisited, providing a minimally invasive alternative for treating renal stones. Currently, laparoscopic pyelolithotomy has been found to be feasible for treating non-staghorn renal calculi, but is limited in its use for relatively capacious extra-renal pelves $[1,2]$. The advent of robotic assistance, with Endowrist technology allowing a full range of movement akin to the hand, has extended the possible use of this technology for treatment of renal stones, especially given the versatility and success of robotic assistance in performing complex renal ablative and reconstructive procedures (pyeloplasty, partial nephrectomy, and donor nephrectomy) [3-5]. We present our experience with robotic extended pyelolithotomy, highlighting our technical variation.

\section{Patients and methods}

We performed "purely" robotic extended pyelolithotomy in three cases. Pre-operative evaluation included a routine urine analysis, renal function test, SMA 20, and an intra-venous urogram to assess the stone burden, shape, and number of stones. Particular note of the renal pelvicalyceal anatomy was made with regard to extension of stone into the infundibulae, the configuration of the renal pelvis (degree of intra/extrarenal component), and the degree of hydronephrosis. All three patients had a unilateral (two right and one left) partial staghorn calculus. Two cases, additionally, had small secondary calculi in the inferior and middle calyces.

Technique

After catheterization, the patients were placed in a lateral decubitus position with minimal side flexion of the 
operating table without elevating the kidney rest. The catheter is kept clamped to allow distension of the urinary bladder, which facilitates antegrade placement of the JJ stent subsequently, as the lower end has greater space to coil. The reflux of fluid via the stent (seen as drops of water emanating from the holes and the end of the stent) is further reassuring regarding correct placement of the lower end of the stent in the bladder, and not in the juxta-vesical ureter.

\section{Port placement}

The pneumoperitoneum is established using the Veress needle by placing it in the ipsilateral hypochondrium/iliac fossa. We avoid placing it at the umbilicus as the patient is already positioned in lateral flank position, which would place the displaced small bowel at risk of injury. The camera port is placed through the lateral edge of the rectus muscle at the level of the umbilicus, while the two 8-mm robotic ports are placed in the midline, between xiphisternum and umbilicus, and the other in the ipsilateral iliac fossa, at least $7-8 \mathrm{~cm}$ away from the camera port, to form a wide isosceles triangle, allowing clash-free excursions of the robotic arms (Fig. 1). The daVinci-S robot is then docked. A 5-mm assistant port is placed in the midline inferiorly, between the umbilicus and pubic bone.

Peritoneoscopy, colon mobilization, and dissection of the renal pelvis

The procedure is initiated, using a $30^{\circ}$ downward facing lens, by a limited mobilization of the colon overlying the kidney and renal pelvis. The ureter is located and traced

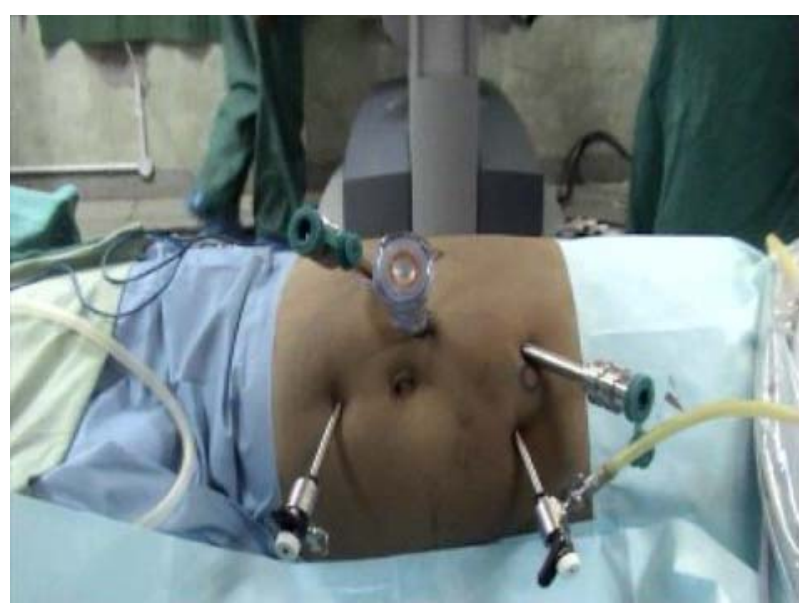

Fig. 1 Port position for right robotic pyelolithotomy after creation of the pneumoperitoneum

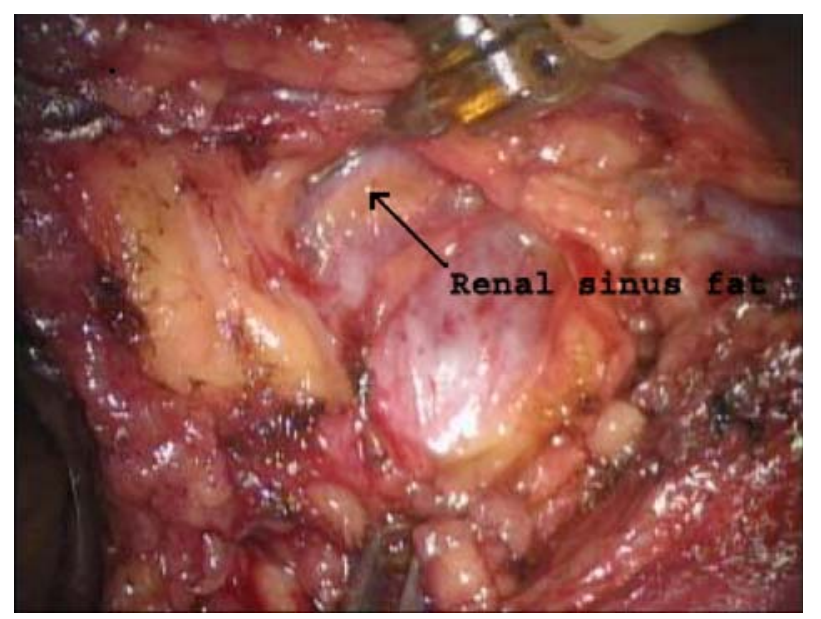

Fig. 2 Dissection of intra-renal pelvis. Renal pelvis (below) and renal parenchyma (above) are seen separated by the renal sinus fat (arrow)

cranially to identify the renal pelvis. Due to the intra-renal location of the pelvis in all patients, careful dissection into the Gil-Vernet's plane was mandatory. In view of a transperitoneal approach, the renal vessels were found to lie abutting the cranial edge of the renal pelvis, and one needs to be careful while dissecting. We did not sacrifice the gonadal vein. Dissection is performed using a Maryland bipolar forceps on the left side and a curved scissor on the right.

\section{Pyelotomy, infundibulotomy, and removal of stones}

Once the pelvis is adequately dissected (Fig. 2), a $\mathrm{V}$-shaped pyelotomy incision is made. The "hook" instrument is utilized in dissecting the pelvic mucosa off the stone, freeing it to allow the stone to be maneuvered into a position such that its least diameter aligns with the pyelotomy. Additionally the pyelotomy was extended into the superior and inferior calyces or their infundibula (Fig. 3). This invariably led to delivering one end of the partial staghorn out first, allowing manipulation of the other end. The inferior and middle calyceal (secondary) calculi were retrieved under vision using Maryland bipolar forceps. The calyces were flushed with saline, directed through an irrigation-suction device. The laparoscopic assistant, using a long-tipped grasping forceps, can also help in removing the secondary calyceal stones.

\section{Antegrade stenting}

An antegrade JJ stent was placed in all cases (Fig. 4). The guide wire was introduced through the 5-mm laparoscopic port. 


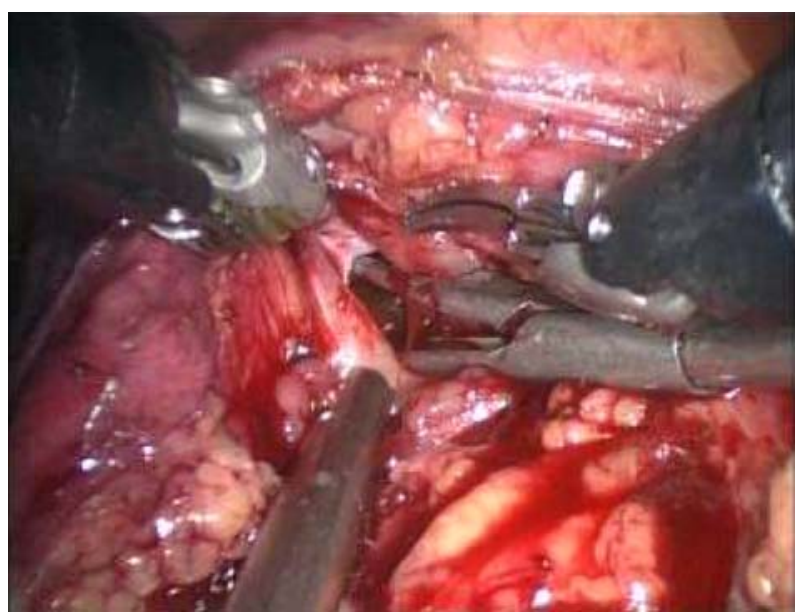

Fig. 3 Infundibulotomy for inferior calyceal secondary calculus in right kidney

Repair of the infundibular and pyelotomy incisions

The infundibular and pyelotomy incisions were sutured using 4-0 Vicryl suture in an interrupted fashion. The Gerota's fascia was also approximated to close off the perinephric space from the peritoneal cavity. An intraperitoneal $14 \mathrm{G}$ drain was placed through 5-mm assistant port.

\section{Retrieval of stones from the body}

The stones were retrieved using a "home-made" plastic bag (Fig. 5) to save on the cost of an Endocatch bag. The robot was de-docked and a $30^{\circ}$ telescope was placed through the 5-mm assistant's port to provide laparoscopic vision. The plastic bag was retrieved by marginally enlarging the $12-\mathrm{mm}$ camera port site, thus avoiding

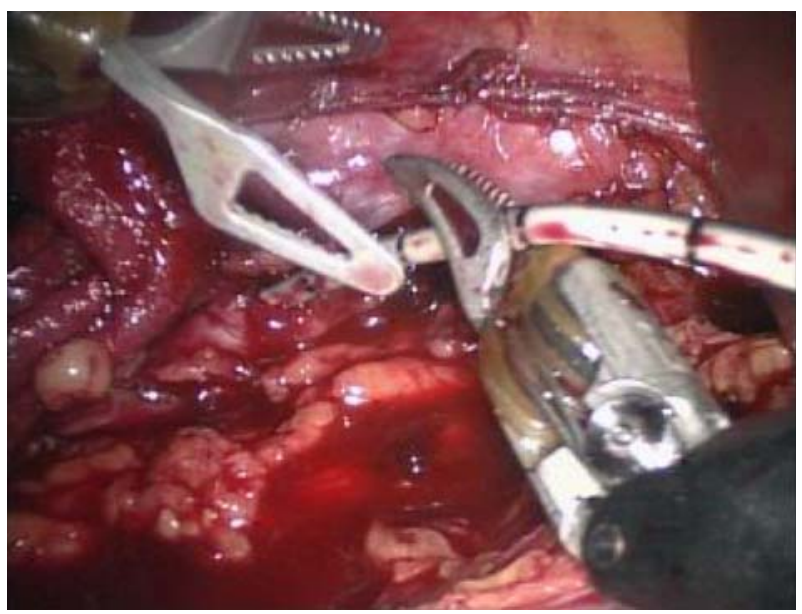

Fig. 4 Antegrade DJ stent placement (see text)

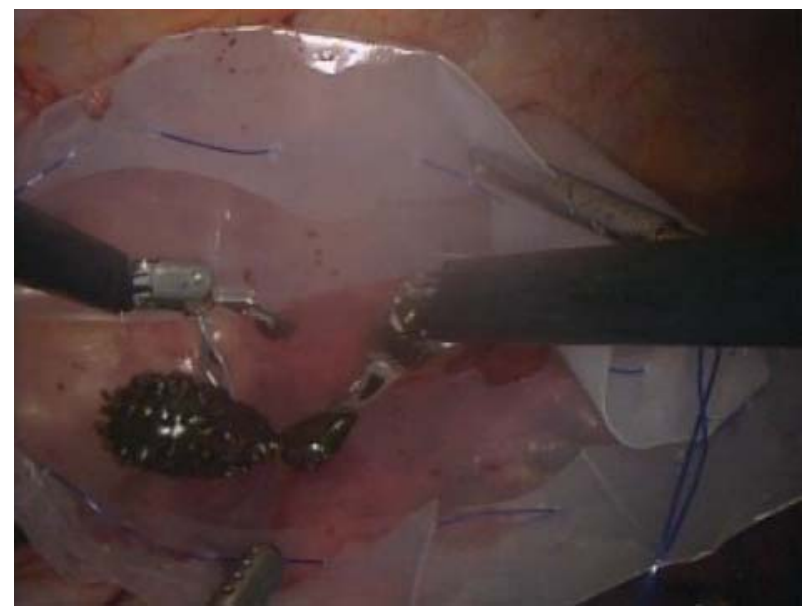

Fig. 5 Home-made plastic bag for retrieval of stones

another incision to remove the bag from the peritoneal cavity. In one case we crushed the stone within the plastic bag using a Kelly's clamp, to facilitate extraction of the bag without unduly enlarging the port site. This way only three robotic ports and an additional 5-mm assistant port are required.

\section{Results}

The procedure was successfully completed "purely" using the robot with a mean operative time of $85 \mathrm{~min}$ and blood loss of less than $50 \mathrm{~cm}^{3}$. The mean stone size was $3.5 \mathrm{~cm}$ (longest diameter). The stones were delivered intact from the extended pyelotomy and the secondary calculi were removed using a combination of the precise bipolar forceps/grasping forceps and saline flush technique. A postoperative X-ray confirmed the position of the JJ stent and documented complete clearance. Post-op recovery was uneventful. The drain was removed on the second post-op day (drain output $<30 \mathrm{~mL}$ ) and patients were discharged on the third post-operative day. The stent was removed four weeks post-operation.

\section{Discussion}

Percutaneous nephrolithotripsy (PNL) is the mainstay treatment for large renal calculi, especially complete staghorn calculi, with or without adjunctive shock wave lithotripsy (SWL). It has replaced the erstwhile open technique of extended pyelolithotomy due to its minimally invasive nature. However, as a procedure it is associated with its own set of technical challenges and complications, especially when treating staghorn renal calculi [6]. Achieving complete clearance may require multiple tracts, 
which increases the risk of renal parenchymal injury. Bleeding and pseudoaneurysm formation is also an incessant risk [7]. Use of a frequently required supracostal tract places the patient at risk of pleural injury occasionally requiring chest tube drainage. Unfavorable pelvicalyceal anatomy with tightly packed calculi and minimal hydronephrosis make tract access difficult. Very large stones prolong the procedure, increasing the risk of fluid absorption and even hypothermia [8]. The association of staghorn calculi with infection also places the patient at risk of sepsis, with stone fragmentation contributing to release of microbes from the stone interstitium. In addition, the procedure requires pre-placement of a ureteral access catheter and iodinated contrast instillation. Stone removal demands use of intra-corporeal energy to achieve stone fragmentation and thus the attendant risk of residual stone fragments.

Laparoscopic pyelolithotomy, both transperitoneal and retroperitoneal, has reportedly shown good results, especially for non-staghorn calculi [9]. Successful laparoscopic stone retrieval performed concomitant with pyeloplasty has also been reported and is much easier than performing an extended pyelolithotomy [10,11]. The advent of robotic assistance and its ability to translate the surgeon's wrist and finger movements precisely in $3 \mathrm{D}$ and in a magnified environment has added a newer dimension in extending the scope of laparoscopy in treating renal stones. Robotic assistance makes it technically easier to perform an extended pyelo-infundibulotomy in a manner similar to that performed during open extended pyelolithotomy. Menon et al. are credited for exploring the feasibility of robotic extended pyelolithotomy with good results suggesting it as a possible alternative to PNL for incomplete (partial) staghorn calculi [4]. In our experience also, robotic assistance allows removal of partial staghorn renal stones even in patients with a predominantly intra-renal pelvis. Additionally it provides all the inherent benefits of extended pyelolithotomy as it avoids renal parenchymal transgression especially when dealing with pelvic bulky renal calculi in solitary kidneys/compromised renal function. Since the stones are removed intact from the kidney, it may minimize the risk of systemic sepsis by preventing release of microbes from inside the stone substance, in infected stones, and also does not leave the fragments inside pelvicalyceal system, thus preventing possible recurrence due to "clinically insignificant residual fragments".

A few technical modifications incorporated by us include:

1 Limited mobilization of the segment of the colon overlying the renal pelvis.

2 Dislodging and manipulating the stone, using a robotic hook, into a position such that the stone could be extracted with its smallest diameter lying parallel to the pyelo-infundibulotomy. This prevents irregular tearing of the pyelotomy.

3 Once a sufficient pyelo-infundibulotomy is made, the stone is held with a precise bipolar forceps or with the assistant grasping forceps and kept under gentle traction with rotatory movements. This maneuver helps in gently pulling out the intra-renal part of the stone under vision, ensuring disimpaction from the mucosa. It also gently pulls out the mucosa of the pelvicalyceal system which gets laid over the stone and makes further extension of the pyelotomy into the intra-renal pelvis or infundibulum much easier and in a more controlled fashion.

4 A 5-mm port was utilized to serve as the assistants' port which subsequently was used for providing laparoscopic view (with a 5-mm telescope) allowing stone retrieval through the camera port $(12 \mathrm{~mm})$ similar to that reported by us while performing minimally invasive retroperitoneoscopic ureterolithotomy [12].

5 In one case where the stone was very large and was not possible to retrieve through the camera port $(12 \mathrm{~mm})$, it was fragmented within the "home-made" plastic bag, to facilitate its removal without enlarging the $12-\mathrm{mm}$ port site.

Robotic pyelolithotomy is however no panacea for treating stone disease and has limitations. It currently entails a transperitoneal/anterior approach to the renal pelvis making the superior extension of the pyelotomy incision difficult due to the presence of the renal vessels. Additionally, it is difficult to provide fluoroscopic control during the procedure. The lack of haptic feedback, unlike open surgery, makes it difficult to perform a nephrotomy over a trapped calyceal stone. Retrieval of secondary stones, if not directly visualized despite "peering" into the calyceal system, becomes an imprecise "blind" procedure. The cost is currently still prohibitive in utilizing this technology.

Despite these drawbacks robotic extended pyelolithotomy appears to be a safe and feasible, minimally invasive, alternative to PNL in carefully selected patients with pelvic bulky partial staghorn calculi.

\section{References}

1. Hemal AK, Goel A, Kumar M, Gupta NP (2001) Evaluation of laparoscopic retroperitoneal surgery in urinary stone disease. $\mathrm{J}$ Enodourol 15:701-705

2. Goel A, Hemal AK (2003) Evaluation of role of retroperitoneoscopic pyelolithotomy and its comparison with percutaneous nephrolithotripsy. Int Urol Nephrol 35(1):73-76

3. Phillips CK, Taneja SS, Stifelman MD (2005) Robot assisted laparoscopic partial nephrectomy: the NYU technique. J Endourol 19:441-445 
4. Badani KK, Hemal AK, Fumo M et al (2006) Robotic extended pyelolithotomy for treatment of renal calculi: a feasibility study. World J Urol 24:198-201

5. Gettman MT, Neurerer R, Bartsch G, Peschel R (2002) Anderson-Hynes dismembered pyeloplasty performed with the $\mathrm{Da}$ Vinci robotic system. Urology 60:509-513

6. Al-Kohlany KM, Shokeir AA, Mosbah A et al (2005) Treatment of complete staghorn stones: a prospective randomized comparison of open surgery versus percutaneous nephrolithotomy. J Urol 173(2):469-473

7. Gremmo E, Ballanger P, Dore B, Aubert J (1999) Hemorrhagic complications during percutaneous nephrolithotomy. Retrospective studies of 772 cases. Prog Urol 9(3):460-463
8. Kukreja RA, Desai MR, Sabnis RB, Patel SH (2002) Fluid absorption during percutaneous nephrolithotomy. Does it matter? J Endourol 16(4):221-224

9. Gaur DD, Agarwal DK, Purohit KC, Darshane AS (1994) Retroperitoneal laparoscopic pyelolithotomy. J Urol 151:927-929

10. Ramakumar S, Lancini V, Chad DY et al (2002) Laparoscopic pyeloplasty with concomitant pyelolithotomy. J Urol 167:13781380

11. Nambirajan T, Jeschke S, Albqami N et al (2005) Role of laparoscopy in management of renal stones: single-center experience and review of literature. J Endourol 19:353

12. Hemal AK, Goel A, Goel R (2003) Minimally invasive retroperitoneoscopic ureterolithotomy. J Urol 169(2):480-482 